\title{
TUBERCULOSIS
}

\section{Isolation prevalence of pulmonary non-tuberculous mycobacteria in Ontario, 1997-2003}

\author{
Theodore K Marras, Pamela Chedore, Alicia M Ying, Frances Jamieson
}

Thorax 2007;62:661-666. doi: 10.1136/thx.2006.070797

See end of article for authors' affiliations

Correspondence to: Dr T K Marras, Toronto Western Hospital, 7E-452, 399 Bathurst Street, Toronto, Ontario, M5T 2S8, Canada; ted.marras@utoronto.ca

Received 23 August 2006 Accepted 1 February 2007 Published Online First 20 February 2007 berculous mycobacteria (NTM) infection is reportedly Background: The prevalence of pulmonary non-tuberculous mycobacteria (NTM) infection is reportedly
increasing. A study was undertaken of the "isolation prevalence" of pulmonary NTM in Ontario, Canada between 1997 and 2003 and the frequency of pulmonary NTM "disease".

Methods: In a retrospective cohort, the "isolation prevalence" was studied by reviewing all positive NTM culture results from the Tuberculosis and Mycobacteriology Laboratory, Ministry of Health and Long-Term Care in Toronto from 1997 to 2003. This laboratory identifies at least 90\% of NTM isolates in Ontario, Canada. Prevalence was compared between years using a negative binomial model. To study the frequency of "disease" (defined by American Thoracic Society criteria), the records of patients who had an isolate in 2003 and had been assessed at our hospital were reviewed.

Results: 22247 pulmonary isolates were obtained from 10231 patients. The "isolation prevalence" of all species (excluding Mycobacterium gordonae) was 9.1/100 000 in 1997, rising to 14.1/100 000 by 2003 $(p<0.0001)$ with a mean annual increase of $8.4 \%$. Similar increases were observed for individual species. 200 patients assessed at our institution were studied using American Thoracic Society criteria for "disease". Microbiological criteria were fulfilled by $37 \%$. Of patients with adequate data, $74 \%$ fulfilled clinical criteria, $77 \%$ fulfilled radiological criteria and $33 \%$ fulfilled all criteria.

Conclusions: The "isolation prevalence" of pulmonary NTM has significantly and rapidly increased in Ontario; a sizeable proportion of patients are likely to have "disease".
$\mathrm{P}$ ulmonary non-tuberculous mycobacteria (NTM) infection can cause severe progressive illness which may be preceded by a period of colonisation. ${ }^{12}$ Treatment of pulmonary NTM is complicated, requiring multiple antimycobacterial drugs for $>12$ months. ${ }^{12}$ Treatment is usually initiated in the setting of "disease", defined by the American Thoracic Society (ATS) as the presence of symptoms, radiographic abnormalities and multiple positive cultures (box 1). ${ }^{1}$ Colonisation, on the other hand, may be defined by the absence of one or more diagnostic criteria. ${ }^{3}$ In the setting of colonisation, treatment is often withheld owing to its potential toxicities and the uncertain rate of progression. Ideally, treatment should be initiated before irreversible lung damage occurs or progresses.

Studies of the epidemiology of pulmonary NTM infections are generally limited in several ways. First, since NTM are often isolated from the environment including potable water, specimen contamination is a significant concern. ${ }^{12}$ Second, the mere presence of NTM in the lungs does not necessarily imply significant or progressive pulmonary disease. ${ }^{22}$ This has resulted in the confusing terminology whose definition is critical to the interpretation of studies. Third, because the isolation of NTM does not mandate informing a "reportable disease" office in many jurisdictions, surveys of the epidemiology of NTM often consist of incomplete samples. Although all epidemiological studies of pulmonary NTM including those referenced in this paper) have major limitations, it seems clear that the prevalence of pulmonary NTM varies by geographical region and is increasing significantly. All well-defined population-based studies of temporal trends of pulmonary NTM consistently report increases in frequency of 1.5-6-fold over 530 years. ${ }^{4-9}$ The universally observed increases in frequency of pulmonary NTM infection makes this emerging infectious disease relevant to all pulmonary and infectious disease practitioners. The precise magnitude of the problem is not well understood for several reasons. In addition to the limitations outlined above, increasing prevalence and geographical variability make studying this infection challenging. Changing prevalence requires frequent study and geographical variability limits generalisability of results of studies across regions. In addition, many previous studies have been limited by representing either small or inadequately defined areas and populations, or incomplete sampling of large and varying areas and populations. ${ }^{10}{ }^{11}$ There is a lack of recent and comprehensive data regarding the frequency of NTM infection in Canada and Ontario. Furthermore, the proportion of patients with colonisation versus disease remains even less well studied. In a systematic review, median annual rates of colonisation in previous studies were 4.4 per 100000 population in North America, 7 per 100000 in Europe and 5.4 per 100000 in Australia. ${ }^{10}$ The corresponding median rates of disease were 1.8 per 100000 in North America, 1.3 per 100000 in Europe and 1.8 per 100000 in Australia. ${ }^{10}$ We studied the "isolation prevalence" of pulmonary NTM in Ontario and examined whether the prevalence changed between 1997 and 2003. In a secondary analysis, we studied a subset of patients to determine the proportion with "disease".

\section{METHODS}

To study the isolation prevalence of pulmonary NTM in Ontario, data were obtained retrospectively from the TB and Mycobacteriology Laboratory of the Ministry of Health and Long-Term Care in Toronto ( $\mathrm{MOH}$ TB laboratory). The number of people with a positive pulmonary NTM culture between 1997 and 2003 was determined. Owing to data constraints, we were unable to study the total number of isolates per person for all years. The study period began in January 1997 when high performance liquid chromatography was introduced in the

Abbreviations: ATS, American Thoracic Society; NTM, non-tuberculous mycobacteria 
Box 1 Definitions of terms regarding pulmonary non-tuberculous mycobacteria (NTM)

Isolation prevalence:

- In a population the prevalence of the isolation of NTM from pulmonary specimens, regardless of clinical status or chest imaging findings of the individual population members

Colonisation:

- Recovery of NTM from pulmonary specimens, and

- Absence of clinical and/or radiographic evidence of infection with important effects

Disease (synonymous with advanced infection, see table 1):

- Repeated recovery of NTM from pulmonary specimens, and

- Progressive clinical signs or symptoms compatible with NTM infection not explained by another illness, and

- Chest imaging abnormalities consistent with NTM infection of the lung parenchyma

mycobacteriology laboratory, providing an accurate species identification method for the most common NTM species. The study period ended in December 2003, the last year for which data were complete when we initiated this study. The Toronto area $\mathrm{MOH}$ TB laboratory processes the majority of specimens for mycobacterial culture in Ontario and also acts as a reference laboratory for species identification for cultures from most other laboratories in the province. We communicated with laboratory directors and senior technologists from other Ontario laboratories that identify NTM organisms to estimate the fraction of provincial NTM isolates that are identified in the Toronto MOH TB laboratory. During the last 5 years of the study (1999-2003) there were approximately 200 isolates per year in other laboratories. Based on our results, we therefore estimate that at least $90 \%$ of NTM isolates in Ontario are identified in the Toronto MOH TB laboratory. We therefore decided that excluding isolates from other laboratories would not significantly alter frequency estimates and included data only from the Toronto area MOH TB laboratory.

To explore the frequency of disease among individuals with pulmonary NTM, we studied a subgroup of patients with pulmonary NTM isolates in 2003. Among patients with pulmonary NTM isolates, the records of those who had been assessed at our hospital network-the University Health Network (an academic institution providing ambulatory and inpatient care)-within 6 months of sample collection were reviewed.

Specimen culture was performed using the Bactec $460 \mathrm{~TB}$ system until May 2000 and thereafter with the Bactec MGIT 960 system which has been shown to be slightly more sensitive for NTM $^{12}$ (Becton Dickinson Microbiology Systems, Sparks, Maryland, USA). Specimens were processed and analysed as described by Hanna et al. ${ }^{12}$ NTM cultures were speciated by DNA probes (AccuProbe, Gen-Probe, San Diego, California, USA) for Mycobacterium avium complex and $M$ gordonae, and by high performance liquid chromatography for other species. ${ }^{13}$

The isolation prevalence of pulmonary NTM was calculated for each of the study years as follows. A prevalent case was defined as a patient with an isolate during a given calendar year (isolation prevalence $=$ total number of patients with an isolate during a given year/contemporary provincial population). To study whether isolation prevalence changed over time, isolation prevalence was compared between years by visually inspecting graphs and statistical significance was assessed using generalised linear models with a negative binomial distribution using prevalence as the outcome variable and year as the predictor variable. This was performed using the SAS Proc Genmod with a negative binomial distribution and a logarithmic link function. Regression was also performed with the SAS Proc Autoreg to verify a lack of autocorrelation.

The above calculations were repeated for the four most commonly isolated species or species groups in each year, excluding $M$ gordonae. Although a common isolate, $M$ gordonae was excluded as it may be a contaminant from water ingested before collecting sputum and is infrequently associated with clinically significant disease. ${ }^{14}$ The prevalence of NTM was compared with the contemporary Ontario tuberculosis case rates.

The proportion of patients with disease was determined only for members of the subset who had been patients at our hospital within 6 months of isolation of NTM. These patients' records were reviewed to determine whether they fulfilled ATS clinical and radiographic criteria for disease (table 1). ${ }^{1}$ Clinical criteria were assessed from clinic and inpatient records. Radiographic criteria were assessed using radiologists' reports.

Data were entered directly into an electronic database in Microsoft Access 2000 (Redmond, Washington, USA). All statistical analyses were performed using SAS Version 8.02 (SAS Institute, Cary, North Carolina, USA) and Epi Info 2000 (Centers for Disease Control and Prevention, Atlanta, Georgia, USA). Ethics approval was granted by the appropriate institutional review board.

\section{RESULTS}

Between 1997 and 2003 there were 23823 NTM isolates from 11186 patients including all body sites. Limiting specimens to pulmonary sites yielded 22247 isolates from 10231 patients. $93 \%$ of all NTM isolates were from a pulmonary source and $91 \%$ of all patients with NTM isolates had specimens from a pulmonary source. Table 2 presents the number of isolates and individuals by anatomical site per year.

Of the $7 \%$ of isolates that were non-pulmonary, 59\% (924/ 1576) were of unknown origin while the remainder were from blood $(37 \%, 590 / 1576)$, urine $(4 \%, 60 / 1576)$, and bone marrow $(0.1 \%, 2 / 1576)$. Of the $9 \%$ of patients who had non-pulmonary isolates, $85 \%(807 / 955)$ of isolates were of unknown origin while the remainder were from blood $(12 \%, 112 / 955)$, urine $(4 \%, 35 / 955)$ and bone marrow $(0.1 \%, 1 / 955)$. The number of patients with pulmonary isolates increased each year with the exception of the final year of the study. Conversely, the number of patients with isolates not known to be pulmonary tended to decrease during the study period, including the number of patients with isolates from blood, urine and unknown anatomical sources. $M$ avium complex was isolated in 59\% of cases, $M$ xenopi was isolated in $26 \%$ of cases, rapidly growing mycobacteria ( $M$ abscessus, $M$ chelonae and $M$ fortuitum) were isolated in $13 \%$ and $M$ kansasii was isolated in $2 \%$ of cases.

Isolation prevalence and temporal trends of pulmonary NTM, along with the contemporary provincial rates of tuberculosis, are shown in table 3. The isolation prevalence for the four most common NTM species combined was 9.1/100 000 in 1997 and rose to $14.1 / 100000$ by 2003 , a statistically significant increase $(\mathrm{p}<0.0001)$ with a mean annual increase of $8.4 \%$. Similar increases in prevalence, all statistically significant, were observed for each individual NTM species (table 3 ).

To investigate whether the increase in isolation prevalence was due to a surveillance bias, we analysed the frequency of 
Table 1 American Thoracic Society diagnostic criteria for pulmonary NTM disease*

\begin{tabular}{|c|c|}
\hline Category & Criteria \\
\hline Clinical & $\begin{array}{l}\text { Compatible signs/symptoms (cough, fatigue, fever, } \\
\text { weight loss, haemoptysis, dyspnoea), documented } \\
\text { deterioration in clinical status and reasonable exclusion of } \\
\text { other disease }\end{array}$ \\
\hline \multirow[t]{2}{*}{ Radiographic } & $\begin{array}{l}\text { On chest radiograph, any of: } \\
\text { infiltrates (progressive or persistent } \geqslant 2 \text { months) } \\
\text { cavitation } \\
\text { multiple nodules }\end{array}$ \\
\hline & $\begin{array}{l}\text { On high resolution computed tomographic scan, any of: } \\
\text { multiple small nodules } \\
\text { multifocal bronchiectasis } \\
\text { (if baseline films }>1 \text { year old, there should be evidence of } \\
\text { progression) }\end{array}$ \\
\hline \multirow[t]{3}{*}{ Microbiological } & $\begin{array}{l}\text { With at least three available sputum/bronchial wash } \\
\text { samples in } 1 \text { year, any of: } \\
\text { three positive cultures } \\
\text { two positive cultures with any acid-fast bacilli seen on at } \\
\text { least one smear }\end{array}$ \\
\hline & $\begin{array}{l}\text { With only one available bronchial wash and inability to } \\
\text { obtain sputum, any of: } \\
\text { positive culture with } 2+, 3+\text { or } 4+\text { growth } \\
\text { positive culture with more than rare acid-fast bacilli seen } \\
\text { on smear assessment }\end{array}$ \\
\hline & $\begin{array}{l}\text { With a tissue biopsy: } \\
\text { any growth on bronchopulmonary tissue biopsy } \\
\text { granuloma and/or acid-fast bacilli on lung biopsy with at } \\
\text { least } 1+\text { growth from sputum/bronchial wash }\end{array}$ \\
\hline
\end{tabular}

NTM, non-tuberculous mycobacteria.

*A patient must meet clinical, radiographic and microbiological criteria to be classified as having pulmonary NTM disease according to American Thoracic Society guidelines.

microscopy smear-positive cases in each year. We reasoned that surveillance bias was probably not important if there was no change in the fraction of cases that were smear-positive. The frequency of smear-positive specimens ranged from a low of $10.5 \%$ in 1997 to a high of $17.4 \%$ in 2000 and was $11.1 \%$ in 2003. There was no obvious temporal trend in the fraction of smear-positive cases. If smear-positive status is a reasonable surrogate for NTM disease, then the relatively stable fraction of cases with smear-positive isolates over time suggests that the frequency of NTM disease probably increased during the study period.

Isolation prevalence of pulmonary NTM increased while the incidence of tuberculosis in Ontario slowly and progressively decreased. The isolation prevalence of individual NTM species, all NTM species combined and the contemporary rates of tuberculosis are shown in fig 1 . The isolation prevalence of pulmonary NTM was greater than the rate of tuberculosis throughout the study period, and the ratio of NTM isolation prevalence to tuberculosis case prevalence increased during the study period.

Of 2090 patients with pulmonary NTM isolates (other than $M$ gordonae) in 2003, $200(10 \%)$ were assessed using ATS diagnostic criteria for disease based on at least one of a clinical or radiographic assessment at our hospital. Isolate sources were sputum in 171 patients $(85.5 \%)$ and by bronchoscopy in 29 patients (14.5\%). NTM species included $M$ avium complex in $59 \%, M$ xenopi in 33.5\%, rapidly growing mycobacteria in $6.5 \%$ and $M$ kansasii in $1 \%$. ATS microbiologic criteria were fulfilled by $73 / 200(37 \%)$ of patients. Adequate clinical information was available for 139 patients, 103 of whom (74\%) fulfilled clinical criteria. Adequate radiological information was available for 180 patients, 139 of whom (77\%) fulfilled radiological criteria. Adequate information for all ATS criteria was available for 119 patients, 39 of whom (33\%) fulfilled all criteria.

\section{DISCUSSION}

We observed a high and increasing isolation prevalence of pulmonary NTM in Ontario, Canada between 1997 and 2003. Based on a systematic review ${ }^{10}$ and more recent work, ${ }^{17}$ the median rates of pulmonary NTM isolation have been approximately 6.2/100 000 in North America, 8.3/100 000 in Europe, 15/100 000 in Asia and 7.2/100 000 in Australia. Our results represent a considerably higher prevalence than is generally reported in North American studies and studies from most other areas. Throughout the period of our study the isolation prevalence of pulmonary NTM was approximately twice as great as the rates of tuberculosis cases (mean isolation prevalence of pulmonary NTM 12.6 versus 6.0 for tuberculosis case rates). ${ }^{15} 16$ In addition to the high isolation prevalence, the increase over time is an important observation that is consistent with previous studies. All well-defined population-based studies that presented data on the frequency of pulmonary NTM isolation or disease over time have shown increases. ${ }^{4-9}$ The numerous reports of increasing frequency are consistent with our results and suggest that pulmonary NTM infection is a growing problem. Increases in the frequency of pulmonary NTM throughout the world have not been adequately explained but there are several possible contributing factors. The increase could either be spurious or smaller than perceived if increased awareness has led to more investigations or improved laboratory methods have led to isolation and identification of previously unrecognised organisms. Studies have been published that support ${ }^{18}$ and refute ${ }^{19}$ this argument. We believe such factors cannot completely explain the observed increases. Part of the increase we observed could be explained by the greater sensitivity of the Bactec MGIT 960 system. However, this could only explain a portion of the increase for at least two reasons. First, the increase in prevalence began before and

Table 2 Number of non-tuberculous mycobacteria (NTM) isolates and patients with isolates per year by anatomical site

\begin{tabular}{|c|c|c|c|c|c|c|}
\hline \multirow[b]{2}{*}{ Year } & \multicolumn{6}{|c|}{ Number of isolates/patients by anatomical source of specimen } \\
\hline & Pulmonary & Blood & Urine & Bone marrow & Unknown & Total $^{*}$ \\
\hline 1997 & $1658 / 1172$ & $55 / 36$ & $25 / 24$ & $0 / 0$ & $232 / 194$ & $1970 / 1386$ \\
\hline 1998 & $1890 / 1214$ & $32 / 23$ & $4 / 4$ & $1 / 1$ & $285 / 249$ & $2212 / 1433$ \\
\hline 1999 & $2407 / 1537$ & $32 / 20$ & $0 / 0$ & $0 / 0$ & $155 / 143$ & $2594 / 1659$ \\
\hline 2000 & $2519 / 1641$ & $29 / 20$ & $10 / 8$ & $0 / 0$ & $131 / 114$ & $2689 / 1755$ \\
\hline 2001 & $3218 / 2043$ & $25 / 20$ & $1 / 1$ & $0 / 0$ & $105 / 92$ & $3349 / 2126$ \\
\hline 2002 & $3471 / 2319$ & $23 / 14$ & $5 / 4$ & $0 / 0$ & $137 / 114$ & $3636 / 2410$ \\
\hline 2003 & $3200 / 2090$ & $28 / 14$ & $10 / 10$ & $0 / 0$ & $122 / 103$ & $3360 / 2186$ \\
\hline Total $^{*}$ & $22247 / 10231$ & $590 / 112$ & $60 / 35$ & $2 / 1$ & $924 / 807$ & $23823 / 11186$ \\
\hline
\end{tabular}

*Total patients does not equal sum of columns or rows due to patients with isolates from different body sites, multiple species or repeatedly positive cultures over years. 
Table 3 Annual isolation prevalence of pulmonary non-tuberculous mycobacteria (NTM) and reported tuberculosis cases in Ontario, 1997-2003

\begin{tabular}{|c|c|c|c|c|c|c|c|}
\hline \multirow[b]{2}{*}{ Year } & \multirow[b]{2}{*}{$\begin{array}{l}\text { Ontario population* } \\
\text { (thousands) }\end{array}$} & \multicolumn{6}{|c|}{ Prevalence $\llbracket$ (total cases) } \\
\hline & & $\begin{array}{l}\text { M avium } \\
\text { complex }\end{array}$ & M xenopi & $\begin{array}{l}\text { Rapidly growing } \\
\text { mycobacteriat }\end{array}$ & M kansasii & All speciesł & Tuberculosis§ \\
\hline 1997 & 11168.4 & $5.6(629)$ & $2.6(293)$ & 1.2 (134) & $0.09(10)$ & $9.1(1020)$ & $6.9(776)$ \\
\hline 1998 & 11336.0 & $6.1(688)$ & $2.1(241)$ & $1.4(156)$ & 0.17 (19) & $9.4(1064)$ & 6.5 (741) \\
\hline 1999 & 11506.4 & $7.2(824)$ & $3.4(388)$ & $1.4(165)$ & $0.17(20)$ & $11.8(1360)$ & $6.0(696)$ \\
\hline 2000 & 11685.4 & $7.6(892)$ & 3.3 (389) & 1.7 (194) & $0.22(26)$ & $12.5(1465)$ & $6.0(696)$ \\
\hline 2001 & 11897.6 & $9.4(1115)$ & 4.0 (474) & $2.0(240)$ & $0.32(38)$ & $15.0(1780)$ & $5.8(688)$ \\
\hline 2002 & 12096.6 & $9.9(1196)$ & $4.7(571)$ & $2.3(282)$ & 0.35 (42) & 16.5 (1991) & $5.6(677)$ \\
\hline 2003 & 12238.3 & $8.4(1027)$ & $4.2(513)$ & $2.0(240)$ & $0.23(28)$ & 14.1 (1734) & $5.4(657)$ \\
\hline Mean annual change (\%) & N/A & 7.7 & 11.3 & & 23.1 & & -4.0 \\
\hline p Value ${ }^{* *}$ & - & $<0.0001$ & $<0.0001$ & $<0.0001$ & 0.001 & $<0.0001$ & $<0.0001$ \\
\hline
\end{tabular}

Prevalence calculated as the number of cases per 100000 Ontario population in a given year.

N/A, not applicable.

*Values for 1999-2003 from Statistics Canada (http://www.statcan.ca/english/Pgdb/demo02.htm, accessed 2004/10/04); values for 1997 and 1998 estimated by back extrapolation.

† $M$ abscessus, $M$ chelonae and $M$ fortuitum.

$\ddagger$ Data for $M$ avium complex, $M$ xenopi, $M$ fortuitum, $M$ chelonae, $M$ abscessus and $M$ kansasii combined.

$\S$ Total number of tuberculosis cases from Public Health and Epidemiology Reports Ontario; ${ }^{15}{ }^{16}$ rate calculated according to population from Statistics Canada.

- Prevalence of NTM species defined as the isolation prevalence and prevalence of tuberculosis defined according to reported cases of tuberculosis.

${ }^{* *} p$ Value for comparing prevalence over time using generalised linear models with a negative binomial distribution.

continued well after the culture system was changed in June 2000. Second, the greater sensitivity of the new system is expected to increase the total number of positive cultures by less than $7 \%,{ }^{12}$ while the number of prevalent cases we observed increased by more than $30 \%$ between the year before and the year after introducing the new system. In addition, since the same microbiological systems are used to detect $M$ tuberculosis complex and NTM organisms, the concurrent decrease in rates of tuberculosis in Ontario $^{15}$ may argue against an apparent increase in NTM isolation simply due to more sensitive microbiological techniques. It may have been possible that changing referral patterns from other laboratories in Ontario contributed to the observed increase in the prevalence of NTM. If some laboratories had previously performed NTM identification but switched to referring NTM isolates to the Toronto MOH TB laboratory during the study period, then the increase in prevalence may be an overestimate. However, based on a review of referral patterns and the number of isolates from relevant laboratories, changing referral patterns could potentially have

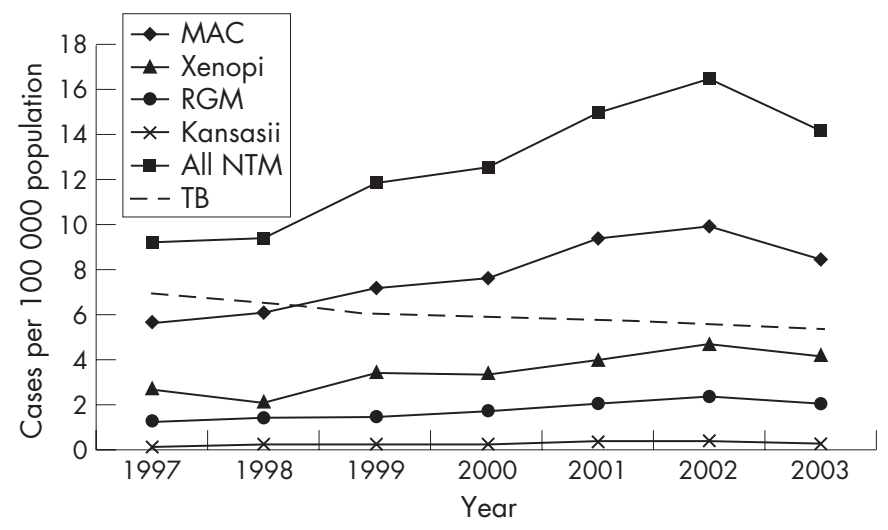

Figure 1 Prevalence of pulmonary non-tuberculous mycobacteria (NTM) infections and tuberculosis (TB) case rates in Ontario 1997-2003. All NTM prevalence rates increased significantly and tuberculosis rates decreased significantly (all $p \leqslant 0.001$ ). Tuberculosis rates calculated from total number of tuberculosis cases ${ }^{15} 16$ and population data from Statistics Canada (for 1999-2003 from http://www.statcan.ca/english/Pgdb/demo02.htm, accessed 2004/10/04) and estimated by back extrapolation (for 1997 and 1998). MAC, M avium complex; RGM, rapidly growing mycobacteria. accounted for an increase of only 3-9\% over the entire study period. Since we observed an increase of over $50 \%$, we believe that changing referral patterns had no important effect on our observation of increased prevalence.

True changes in the frequency of pulmonary NTM isolation could be due to host changes, pathogen changes or an interaction between host and pathogen. Host changes leading to increased susceptibility could play an important role. There may be more immunocompromised patients due to HIV infection, malignancy or simply advanced age. ${ }^{19}$ As proposed elsewhere, changes in host immunity may be relevant. If infections with $M$ tuberculosis complex species ${ }^{20}{ }^{21}$ and BCG $^{22} 23$ provide cross-immunity or protection against pulmonary NTM and if infections with these "protective" mycobacteria decrease, pulmonary NTM may secondarily increase. Decreasing rates of tuberculosis concomitant with increases in pulmonary NTM have generally been observed..$^{20}$ Our observations in Ontario have been similar, with a small progressive concomitant decline in tuberculosis during our study period (table 3, fig 1 ). ${ }^{15}{ }^{16}$ In addition, studies from Sweden ${ }^{23}$ and the Czech Republic ${ }^{22}$ reported that children who were not vaccinated with BCG had a far higher rate of extrapulmonary NTM infection, which is also consistent with the notion that cross-immunity offers protection from infections across mycobacterial species. Pathogen changes such as enhanced virulence should be considered as a possible contributor to increased rates of NTM infection. NTM isolates from patients have been shown to vary widely in virulence. ${ }^{24}$ Examples of genetically identified virulence factors include a conserved coding sequence of a macrophage-induced gene $e^{25}$ and the presence of numerous or large plasmids. ${ }^{26}$ Finally, an interaction between host and pathogen could involve an increase in host exposure to the pathogen. This may have occurred as a result of the increase in popularity of showering as a form of bathing, ${ }^{27}$ a habit that may greatly increase respiratory exposure to water-borne contaminants through aerosolisation. Regardless of the explanation, increasing frequencies of pulmonary NTM are consistently observed which suggests that the importance of pulmonary NTM infection continues to grow.

Mycobacterium xenopi comprised 25-30\% of prevalent cases in our study, a relatively high rate compared with systematic surveys in other parts of North America. ${ }^{4}{ }^{20} 21$ 28-30 This finding is consistent, however, with investigators who have presented 
large series of pulmonary $M$ xenopi infection in southern Ontario $^{3132}$ and northwestern New York State, USA. ${ }^{33}$ We hypothesise that this is related to our proximity to large bodies of fresh water.

In the subset of patients who had adequate clinical information available for review, 33\% met ATS diagnostic criteria for clinically important disease. This is slightly higher than the expected rate of $20-25 \%$ based on the findings of a systematic review of rates of advanced infection among patients with $M$ avium complex or $M$ xenopi recovered from respiratory specimens. ${ }^{10}$ Although the difference may not be significant, a possible explanation for a slightly higher rate could reflect a biased selection of records for review. It is likely that NTMinfected patients assessed at the University Health Network have relatively high rates of disease, given that our institution provides tertiary care. We believe that the results of our subgroup study are applicable to patients seen in a teaching institution that provides primary to tertiary care where NTM isolates may more often signify disease. Further, we note that approximately three-quarters of patients fulfilled clinical and radiological criteria for disease while only one-third fulfilled the criteria we use to define disease. We wonder whether our estimate of the frequency of disease in this sample is a significant underestimate possibly resulting from inadequate microbiological sampling. Unfortunately, data regarding the total number of specimens collected were not available to us so this remains speculative.

The limitations of our study relate to challenges in studying population level epidemiology of the isolation prevalence of pulmonary NTM and challenges in studying the presence of pulmonary NTM disease in a group of patients with pulmonary NTM isolates. For the population-based study of the isolation prevalence of pulmonary NTM, we chose not to study incidence because of the presumed chronic nature of pulmonary NTM. To confidently identify a new or incident case, a long "look-back" period would be required to ensure that a patient had not previously had a pulmonary NTM isolate. The uncertain duration of the "look-back" period required and the lack of reliable data for an adequate duration prior to our study period dissuaded us from studying incidence and we instead focused on prevalence. Looking at individual patients over time, we found that few patients had repeat isolates over years. This observation could be due either to the resolution of the "infection" or infrequent follow-up specimen collection after the initial isolate. Assuming that pulmonary NTM is usually chronic, we suspect that a large proportion of patients did not have regular follow-up specimens collected and, for this reason, the isolation prevalence we observed may be an underestimate. Another limitation in the population-based study is the lack of data from some Ontario laboratories that identify NTM species. We included only isolates from the Toronto MOH TB laboratory. Isolates in other laboratories were not included unless they were referred to the study laboratory so the isolation prevalence of pulmonary NTM in Ontario that we measured is an underestimate. The estimate that our study captured more than $90 \%$ of NTM isolates in Ontario is based on extensive communications with personnel from other laboratories. Although we are confident that this is a fairly accurate estimate and that there was no temporal variation in isolation prevalence in other laboratories that could alter our results, our data remain limited in this area. A third limitation in the population-based study involves specimens that were from an unknown source. In approximately $7 \%$ of specimens the anatomical site of origin was not identified. We suspect that some of these specimens were pulmonary and therefore our results may represent a further underestimate of the isolation prevalence of pulmonary NTM. Overall, we believe that the limitations of the population-based study suggest that our measurements underestimate the isolation prevalence of pulmonary NTM infection and that the true prevalence is higher than we measured.

Our assessment of the frequency of disease among patients with pulmonary NTM isolates may be limited by incomplete data and biased sampling. Incomplete data regarding clinical and radiographic criteria for advanced infection would lead to an underestimate of the frequency of disease. On the other hand, selecting patients assessed at an academic institution probably assembled a cohort of patients with pulmonary NTM isolates who have above average rates of disease. Given that these two biases have opposite effects on the estimated frequency of disease, it is difficult to speculate on the generalisability of our estimate to other populations. We believe that our estimate is valid for patients assessed in a tertiary care centre and that clinicians should strongly consider the possibility that disease is present when pulmonary NTM are isolated in the tertiary care setting.

Our population-based study of the isolation prevalence of pulmonary NTM in Ontario, Canada revealed a higher prevalence than that reported in previous studies. The high and consistently increasing prevalence we observed suggests that the burden of pulmonary NTM infection is significant and will continue to grow in importance. Further work is needed to understand the frequency of disease among all patients with pulmonary NTM. In addition, research efforts should be directed towards areas that will lead to strategies for prevention, predicting progression from the colonised state to disease, and improved treatment of disease.

\section{ACKNOWLEDGEMENTS}

The authors acknowledge the thoughtful reviews by Niall Ferguson, MD and Matthew Stanbrook, MD.

\section{Authors' affiliations}

Theodore K Marras, Alicia M Ying, Joint Division of Respirology, University Health Network and Mount Sinai Hospital, Department of Medicine, University of Toronto, Toronto, Canada

Pamela Chedore, Head TB and Mycobacteriology Laboratory, Public Health Laboratories Branch, Ontario Ministry of Health and Long-Term Care, Toronto, Canada

Frances Jamieson, Clinical and Environmental Microbiology, Public Health Laboratories Branch, Ontario Ministry of Health and Long-Term Care, Toronto, Canada

Ms Ying was an Ontario Thoracic Society studentship awardee.

Competing interests: None.

\section{REFERENCES}

1 American Thoracic Society. Diagnosis and treatment of disease caused by nontuberculous mycobacteria. Am J Respir Crit Care Med 1997;156:S1-19.

2 British Thoracic Society. Management of opportunist mycobacterial infections: Joint Tuberculosis Committee guidelines 1999. Thorax 2000;55:210-8.

3 Larsson LO, Bentzon MW, Lind A, et al. Sensitivity to sensitins and tuberculin in Swedish children. Part 5: A study of school children in an inland rural area. Tuberc Lung Dis 1993;74:371-6

4 Robakiewicz M, Grzybowski S. Epidemiological aspects of non-tuberculous mycobacterial disease and of tuberculosis in British Columbia. Am Rev Respir Dis 1974;109:613-20.

5 DuMoulin GC, Sherman IH, Hoaglin DC, et al. Mycobacterium avium complex, an emerging pathogen in Massachusetts. J Clin Microbiol 1985;22:9-12.

6 Isaac-Renton JL, Allen EA, Chao CW, et al. Isolation and geographic distribution of Mycobacterium other than M tuberculosis in British Columbia, 1972-81. CMAJ 1985; 133:573-6.

7 Debrunner $M$, Salfinger $M$, Brandli $O$, et al. Epidemiology and clinical significance of nontuberculous mycobacteria in patients negative for human immunodeficiency virus in Switzerland. Clin Infect Dis 1992;15:330-45.

8 Kubin M, Svandova E, Medek B, et al. Mycobacterium kansasii infection in an endemic area of Czechoslovakia. Tubercle 1980;61:207-12.

9 Sakatani M. Nontuberculous mycobacteriosis; the present status of epidemiology and clinical studies (in Japanese). Kekkaku 1999;74:377-84. 
10 Marras TK, Daley CL. Epidemiology of human pulmonary infection with nontuberculous mycobacteria. Clin Chest Med 2002;23:553-67.

11 Martin Casabona N, Bahrmand AR, Bennedsen J, et al. Non-tuberculous mycobcteria: patterns of isolation. A multi-country retrospective survey. Int J Tuberc Lung Dis 2004;8:1 186-93.

12 Hanna BA, Ebrahimzadeh A, Elliott LB, et al. Multicenter evaluation of the BACTEC MGIT 960 system for recovery of mycobacteria. J Clin Microbiol 1999;37:748-52.

13 Butler WR, Guthertz LS. Mycolic acid analysis by high-performance liquid chromatography for identification of Mycobacterium species. Clin Microbiol Rev 2001;14:704-26.

14 Arnow PM, Bakir M, Thompson K, et al. Endemic contamination of clinical specimens by Mycobacterium gordonae. Clin Infect Dis 2000;31:472-6.

15 Vik J. Epidemiology of tuberculosis in Ontario, 1999-2001. Public Health and Epidemiology Report Ontario 2003;14:105-15.

16 Ministry of Health and Long-Term Care. Summary of reportable diseases in Ontario - 4th quarter 2003. Public Health and Epidemiology Report Ontario 2004; 15:73-5.

17 Haverkort F. National atypical mycobacteria survey, 2000. Commun Dis Intell 2003;27:180-9.

18 Donnabella V, Salazar-Schicchi J, Bonk S, et al. Increasing incidence of Mycobacterium xenopi at Bellevue Hospital: an emerging pathogen or a product of improved laboratory methods? Chest 2000;1 18:1365-70.

19 Prince DS, Peterson DD, Steiner RM, et al. Infection with Mycobacterium avium complex in patients without predisposing conditions. N Engl J Med 1989;321:863-8.

20 Fogan L. PPD antigens and the diagnosis of mycobacterial diseases. Arch Intern Med 1969; 124:49-54

21 Ahn CH, Lowell JR, Onstad GD, et al. A demographic study of disease due to Mycobacterium kansasii or $M$ intracellulare-avium in Texas. Chest 1979;75:120-5.
22 Trnka L, Dankova D, Svandova E. Six years' experience with the discontinuation of $B C G$ vaccination: 4 . Protective effect of $B C G$ vaccination against the Mycobacterium avium intracellulare complex. Tuberc Lung Dis 1994;75:348-52.

23 Romanus V, Hallander $\mathrm{HO}$, Wahlen P, et al. Atypical mycobacteria in extrapulmonary disease among children. Incidence in Sweden from 1969 to 1990 related to changing BCG vaccination coverage. Tuberc Lung Dis 1995;76:300-10.

24 Ohkusus K, Bermudez LE, Nash KA, et al. Differential virulence of Mycobacterium avium strains isolated from HIV-infected patients with disseminated $M$ avium complex disease. J Infect Dis 2004;190:1347-54.

25 Meyer M, von Grunberg PW, Knoop T, et al. The macrophage-induced gene mig as a marker for clinical pathogenicity and in vitro virulence of Mycobacterium avium complex strains. Infect Immun 1998;66:4549-52.

26 Gangadharam PR, Perumal VK, Jairam BT, et al. Virulence of Mycobacterium avium complex strains from acquired immune deficiency syndrome patients: relationship with characteristics of the parasite and host. Microb Pathog 1989;7:263-78.

27 O'Brien DP, Currie BJ, Krause VL. Nontuberculous mycobacterial disease in Northern Australia: a case series and review of the literature. Clin Infect Dis 2000;31:958-68.

28 O'Brien RJ, Geiter U, Snider DE Jr. The epidemiology of nontuberculous mycobacterial diseases in the United States. Results from a national survey. Am Rev Respir Dis 1987;135:1007-14.

29 Good RC. Isolation of non-tuberculous mycobacteria in the United States, 1979 $J$ Infect Dis 1980;142:779-83.

30 Good RC, Snider DE Jr. Isolation of nontuberculous mycobacteria in the United States, 1980. J Infect Dis 1982;146:829-33.

31 Contreras MA, Cheung OT, Sanders DE, et al. Pulmonary infection with nontuberculous mycobacteria. Am Rev Respir Dis 1988;137:149-52.

32 Simor AE, Salit IE, Vellend H. The role of Mycobacterium xenopi in human disease. Am Rev Respir Dis 1984; 129:435-8.

33 Jiva TM, Jacoby HM, Weymouth LA, et al. Mycobacterium xenopi: innocent bystander or emerging pathogen? Clin Infect Dis 1997;24:226-32.

\section{PULMONARY PUZZLE}

\section{A pleural mass with pulmonary infiltrates}

\section{Clinical presentation}

A healthy 34-year-old woman had a 1-month history of dyspnoea and an abnormal chest radiograph. A course of erythromycin was unhelpful. Further details of her history did not suggest an aetiology. Physical examination showed only mild hypoxia on exertion.

A high-resolution chest CT scan was performed, revealing a left apical and hilar mass extending posteriorly to encase the lung, particularly along the mediastinal aspect (fig 1). There was also bilateral airspace opacification in a bronchocentric distribution. Bronchoscopy and thoracentesis were non-diagnostic.

\section{Question}

What is your diagnosis?

See page 695

This case was submitted by:

C J Ryerson, S Malhotra, S Lam Department of Respiratory Medicine, Vancouver General Hospital, Vancouver, Canada

J C English, D N lonescu

Department of Pathology and Laboratory Medicine, Vancouver General Hospital, Vancouver, Canada

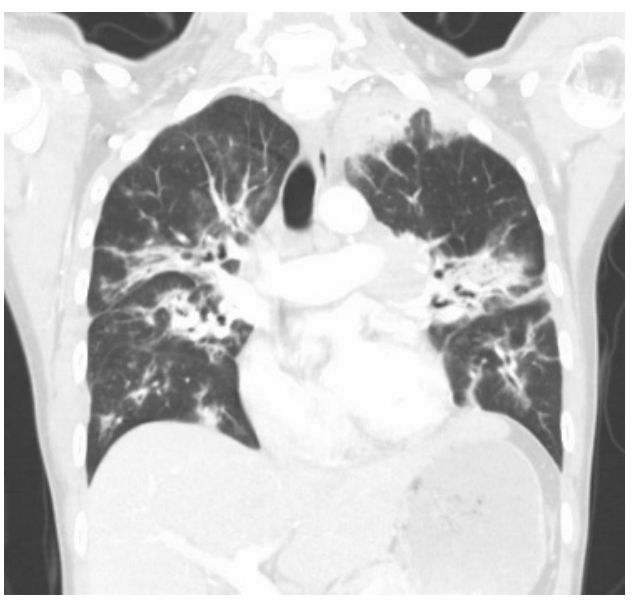

Figure 1 Coronal CT scan of the chest showing ground glass and dense airspace opacification in a bronchocentric distribution throughout both lungs. There is also left hilar enlargement and multiple pleural masses.

Correspondence to: Dr Chris Ryerson, Department of Respiratory Medicine, Gordon \& Leslie Diamond Health Care Centre, 2775 Laurel St, Vancouver General Hospital, Vancouver, Canada V5Z 1M9; cryerson@interchange.ubc.ca

doi: $10.1136 /$ thx.2007.084806 